\title{
Initial study of deep inelastic scattering with ZEUS at HERA
}

ZEUS Collaboration

\author{
M. Derrick, D. Krakauer, S. Magill, B. Musgrave, J. Repond, S. Repond, R. Stanek, \\ R.L. Talaga, J. Thron \\ Argonne National Laboratory, Argonne, IL 60439, USA
}

F. Arzarello, R. Ayad ${ }^{1}$, G. Barbagli ${ }^{2}$, G. Bari, M. Basile, L. Bellagamba, D. Boscherini, A. Bruni, G. Bruni, P. Bruni, G. Cara Romeo, G. Castellini ${ }^{3}$, M. Chiarini, L. Cifarelli, F. Cindolo, F. Ciralli, A. Contin, S. D’Auria, C. Del Papa, F. Frasconi, P. Giusti, G. Iacobucci, G. Laurenti, G. Levi, Q. Lin, B. Lisowski, G. Maccarrone, A. Margotti, T. Massam, R. Nania, C. Nemoz, F. Palmonari, G. Sartorelli, R. Timellini, Y. Zamora Garcia ${ }^{1}$, A. Zichichi University of Bologna and INFN Bologna, 1-40126 Bologna, Italy

A. Bargende, J. Crittenden, H. Dabbous ${ }^{4}$, K. Desch, B. Diekmann, T. Doeker, M. Geerts, G. Geitz, B. Gutjahr, H. Hartmann, J. Hartmann, D. Haun, K. Heinloth, E. Hilger, H.-P. Jakob, S. Kramarczyk, M. Kückes, A. Mass, S. Mengel, J. Mollen, D. Monaldi, H. Müsch, E. Paul, R. Schattevoy, J.-L. Schneider, R. Wedemeyer Physikalisches Institut der Universität Bonn, W-5300 Bonn 1, FRG

A. Cassidy, D.G. Cussans, N. Dyce, H.F. Fawcett, B. Foster, R. Gilmore, G.P. Heath, M. Lancaster, T.J. Llewellyn, J. Malos, C.J.S. Morgado, R.J. Tapper, S.S. Wilson

Bristol University, Bristol BS8 ITL, UK

R.R. Rau

Brookhaven National Laboratory, Upton, NY 11973, USA

T. Barillari, M. Schioppa, G. Susinno

Physics Department, Calabria University, and INFN, I-87036 Cosenza, Italy

A. Bernstein, A. Caldwell, I. Gialas, J.A. Parsons, S. Ritz, F. Sciulli ${ }^{5}$, P.B. Straub, L. Wai, S. Yang Nevis Laboratories, Columbia University, Irvington on Hudson, NY 10533, USA

W. Burkot, A. Eskreys, K. Piotrzkowski, M. Zachara, L. Zawiejski

Institute of Nuclear Physics, Cracow, Poland

P. Borzemski, K. Jeleń, D. Kisielewska, T. Kowalski, E. Rulikowska-Zarębska, L. Suszycki, J. Zając

Institute of Physics and Nuclear Techniques, Academy of Mining and Metallurgy, Cracow, Poland 
T. Kȩdzierski, A. Kotański, M. Przybycień

Department of Physics, Jagellonian University, PL-30059 Cracow, Poland

L.A.T. Bauerdick, U. Behrens, J.K. Bienlein, C. Coldewey, A. Dannemann, K. Dierks ${ }^{4}$, W. Dorth ${ }^{4}$, G. Drews, P. Erhard ${ }^{6}$, M. Flasiński ${ }^{7}$, I. Fleck, A. Fürtjes, R. Gläser ${ }^{8}$, P. Göttlicher, T. Haas, L. Hagge, W. Hain, D. Hasell, H. Hultschig, G. Jahnen ${ }^{9}$, P. Joos, M. Kasemann, R. Klanner, W. Koch, U. Kötz, H. Kowalski, J. Labs, A. Ladage, B. Löhr, M. Löwe, D. Lüke, J. Mainusch, O. Manczak ${ }^{10}$, M. Momayezi, J.S.T. Ng, S. Nickel, D. Notz, I.H. Park, K.-U. Pösnecker ${ }^{11}$, M. Rohde, E. Ros ${ }^{12}$, U. Schneekloth, J. Schroeder, W. Schulz, F. Selonke, E. Stiliaris, E. Tscheslog ${ }^{13}$, T. Tsurugai, F. Turkot ${ }^{14}$, W. Vogel ${ }^{15}$, T. Woeniger ${ }^{16}$, G. Wolf, C. Youngman

Deutsches Elektronen-Synchrotron DESY, W-2000 Hamburg 52, FRG

H.J. Grabosch, A. Leich, A. Meyer, C. Rethfeldt, S. Schlenstedt

Institut für Hochenergiephysik, DESY-Zeuthen, O-1615 Zeuthen, FRG

R. Casalbuoni, S. De Curtis, D. Dominici, A. Francescato, M. Nuti, P. Pelfer University of Florence and INFN, 1-50125 Florence, Italy

G. Anzivino, R. Casaccia, S. De Pasquale, S. Qian, L. Votano

INFN, Laboratori Nazionali di Frascati, I-00044 Frascati, Italy

A. Bamberger, A. Freidhof, T. Poser, S. Söldner-Rembold, G. Theisen, T. Trefzger

Physikalisches Institut der Universität Freiburg, W-7800 Freiburg, FRG

N.H. Brook, P.J. Bussey, A.T. Doyle, J.R. Forbes, V.A. Jamieson, C. Raine, D.H. Saxon Department of Physics and Astronomy, University of Glasgow, Glasgow G12 8QQ, UK

H. Brückmann ${ }^{17}$, G. Gloth, U. Holm, H. Kammerlocher, B. Krebs, T. Neumann, K. Wick I. Institute of Experimental Physics, Hamburg University, W-2000 Hamburg, FRG

A. Hofmann ${ }^{17}$, W. Kröger, J. Krüger, E. Lohrmann, J. Milewski ${ }^{10}$, M. Nakahata, N. Pavel, G. Poelz, R. Salomon ${ }^{18}$, A. Seidman ${ }^{19}$, W. Schott, B.H. Wiik, F. Zetsche

II. Institute of Experimental Physics, Hamburg University, W-2000 Hamburg 50, FRG

T.C. Bacon, I. Butterworth, C. Markou, D. McQuillan, D.B. Miller, M.M. Mobayyen, A. Prinias, A. Vorvolakos

High Energy Nuclear Physics Group, Imperial College London, London SW7 2BZ, UK

T. Bienz, H. Kreutzmann, U. Mallik, E. McCliment, M. Roco, M.Z. Wang

Physics and Astronomy Department, University of Iowa, Iowa City, IA 52240, USA 
P. Cloth, D. Filges

Institut für Kernphysik, Forschungszentrum Jülich, W-5170 Jülich 1, FRG

L. Chen, R. Imlay, S. Kartik, H.-J. Kim, R.R. McNeil, W. Metcalf

Department of Physics and Astronomy, Louisiana State University, Baton Rouge, LA 70803, USA

F. Barreiro ${ }^{20}$, G. Cases, L. Hervás ${ }^{21}$, L. Labarga ${ }^{21}$, J. del Peso, J. Roldán, J. Terrón, J.F. de Trocóniz ${ }^{22}$

Departamento de Física Teóríca, Universidad Autónoma de Madrid, E-28049 Madrid, Spain

F. Ikraiam, J.K. Mayer, G.R. Smith

Department of Physics, University of Manitoba, Winnipeg, Manitoba, Canada R3T 2N2

F. Corriveau, D.J. Gilkinson, D.S. Hanna ${ }^{5}$, L.W. Hung, J.W. Mitchell, P.M. Patel, L.E. Sinclair, D.G. Stairs, R. Ullmann

Department of Physics, McGill University, Montreal, Quebec, Canada H3A 2T8

G.L. Bashindzhagyan, P.F. Ermolov, Y.A. Golubkov, V.A. Kuzmin, E.N. Kuznetsov, A.A. Savin, A.G. Voronin, N.P. Zotov

Institute of Nuclear Pysics, Moscow State University, 117234 Moscow, Russian Federation

S. Bentvelsen, A. Dake, J. Engelen, P. de Jong, S.de Jong ${ }^{12}$, M. de Kamps, P. Kooijman, A. Kruse, H. van der Lugt, V. O'Dell, J. Straver ${ }^{12}$, A. Tenner, H. Tiecke, H. Uijterwaal ${ }^{23}$, J. Vermeulen, L. Wiggers, E. de Wolf, R, van Woudenberg, R. Yoshida

NIKHEF, NL-1009 DB Amsterdam, The Netherlands

B. Bylsma, L.S. Durkin, C. Li, T.Y. Ling, K.W. McLean, W.N. Murray, S.K. Park, T.A. Romanowski ${ }^{24}$, R. Seidlein

Physics Department, Ohio State University, Columbus, OH 43210, USA

G.A. Blair, J.M. Butterworth, A. Byrne, R.J. Cashmore, A.M. Cooper-Sarkar, R.C.E. Devenish, D.M. Gingrich ${ }^{25}$, P.M. Hallam-Baker ${ }^{26}$, N. Harnew, T. Khatri, K.R. Long, P. Luffman, I. McArthur, P. Morawitz, J. Nash, S.J.P. Smith ${ }^{27}$, N.C. Roocroft, F.F. Wilson

Department of Physics, University of Oxford, Oxford, UK

G. Abbiendi, R. Brugnera, R. Carlin, F. Dal Corso, M. De Giorgi, U. Dosselli, F. Gasparini, S. Limentani, M. Morandin, M. Posocco, L. Stanco, R. Stroili, C. Voci

Dipartimento di Fisica dell' Università and INFN, I-35131 Padua, Italy

G. Feild, J.N. Lim, B.Y. Oh ${ }^{28}$, J. Whitmore ${ }^{29}$

Department of Physics, Pennsylvania State University, University Park, PA 16802, USA 
U. Contino, G. D’Agostini, M. Guida ${ }^{30}$, M. Iori, S.M. Mari, G. Marini, M. Mattioli, A. Nigro Dipartimento di Fisica, Università "La Sapienza", I-00185 Rome, Italy

J.C. Hart, N.A. McCubbin, T.P. Shah, T.L. Short

Rutherford Appleton Laboratory, Chilton, Didcot, Oxon OXI1 0QX, UK

E. Barberis, N. Cartiglia, C. Heusch, B. Hubbard, J. Leslie, K. O’Shaughnessy, H.F. Sadrozinski, A. Seiden University of California, Santa Cruz, CA 95064, USA

E. Badura, J. Biltzinger, H. Chaves, M. Rost, R.J. Seifert, A.H. Walenta, W. Weihs, G. Zech Fachbereich Physik der Universität-Gesamthochschule Siegen, W-5900 Siegen 21, FRG

S. Dagan, A. Levy, D. Zer-Zion

School of Physics, Tel-Aviv University, Tel Aviv 69978, Israel

T. Hasegawa, M. Hazumi, T. Ishii, S. Kasai ${ }^{31}$, M. Kuze, Y. Nagasawa, M. Nakao, H. Okuno, K. Tokushuku, T. Watanabe, S. Yamada Institute for Nuclear Study, University of Tokyo, Tokyo 188, Japan

M. Chiba, R. Hamatsu, T. Hirose, S. Kitamura, S. Nagayama, Y. Nakamitsu Department of Physics, Tokyo Metropolitan University, Tokyo 158, Japan

M. Arneodo, M. Costa, M.I. Ferrero, L. Lamberti, S. Maselli, C. Peroni, A. Solano, A. Staiano Dipartimento di Fisica Sperimentale, Università di Torino, and INFN, I-10125 Turin, Italy

\section{Dardo}

Facultá di Scienze MFN, Università di Torino, Alessandria, Italy

D.C. Bailey, D. Bandyopadhyay, F. Benard, S. Bhadra, M. Brkic, B.D. Burow, F.S. Chlebana, M.B. Crombie, G.F. Hartner, G.M. Levman, J.F. Martin, R.S. Orr, J.D. Prentice,

C.R. Sampson, G.G. Stairs, R.J. Teuscher, T.-S. Yoon

Department of Physics, University of Toronto, Toronto, Ontario, Canada M5S IA7

F.W. Bullock, C.D. Catterall, J.C. Giddings, T.W. Jones, A.M. Khan, J.B. Lane, P.L. Makkar, D. Shaw, J. Shulman

Physics and Astronomy Department, University College London, London WC1E 6BT, UK

K. Blankenship, D.B. Gibaut, J. Kochocki, B. Lu, L.W. Mo

Physics Department, Virginia Polytechnic Institute, Blacksburg, VA 24061, USA 
K. Charchuła, J. Ciborowski, J. Gajewski, G. Grzelak, M. Kasprzak, M. Krzyżanowski, K. Muchorowski, R.J. Nowak, J.M. Pawlak, K. Stojda, A. Stopczyński, R. Szwed, T. Tymieniecka ${ }^{32}$, R. Walczak, A.K. Wróblewski, J.A. Zakrzewski, A.F. Zarnecki Institute of Experimental Physics, Warsaw University, PL-00681 Warsaw, Poland

M. Adamus

Institute for Nuclear Studies, Warsaw, Poland

\author{
H. Abramowicz ${ }^{10}$, Y. Eisenberg, C. Glasman, U. Karshon, A. Montag, D. Revel, A. Shapira \\ Nuclear Physics Department, Weizmann Institute, Rehovot 76100, Israel
}

I. Ali, B. Behrens, U. Camerini, S. Dasu, C. Fordham, C. Foudas, A. Goussiou, M. Lomperski, R.J. Loveless, P. Nylander, M. Ptacek, D.D. Reeder, W.H. Smith, S. Silverstein

Department of Physics, University of Wisconsin, Madison, WI 53706, USA

\author{
W.R. Frisken, K.M. Furutani, and Y. Iga \\ Department of Physics, York University, North York, Ontario, Canada
}

Received 21 December 1992

Results are presented on neutral current, deep inelastic scattering measured in collisions of $26.7 \mathrm{GeV}$ electrons and $820 \mathrm{GeV}$ protons. The events typically populate a range in $Q^{2}$ from 10 to $100 \mathrm{GeV}^{2}$. The values of $x$ extend down to $x \sim 10^{-4}$ which is two orders of magnitude lower than previously measured at such $Q^{2}$ values in fixed target experiments. The measured cross sections are in accord with the extrapolations of current parametrisations of parton distributions.

1 Supported by Worldlab, Lausanne, Switzerland.

Present address: INFN, Florence, Italy.

3 Also at IROE, Florence, Italy.

4 Now a self-employed consultant.

5 Present address: DESY, Hamburg, FRG, as Alexander von Humboldt Fellow.

6 Present address: IST GmbH, Darmstadt, FRG.

7 On leave from Jagellonian University, Cracow, Poland.

8 Present address: Martin \& Associates, Hamburg,FRG.

9 Present address: Harry Hoffmann, Fitzbek, FRG.

10 On leave from Warsaw University, Warsaw, Poland.

11 Present address: Lufthansa, Frankfurt, FRG.

12 Present address: CERN, Geneva, Switzerland.

13 Present address: Integrata, Frankfurt, FRG.

14 On leave from FERMILAB, Batavia, IL, USA.

15 Present address: Blohm \& Voss, Hamburg.

16 Present address: BMW, Munich.

17 Deceased.

18 Left II. Institute of Experimental Physics, University of Hamburg, in 1990.
19 On leave from Tel Aviv University, Israel.

20 On leave of absence at DESY, supported by DGICYT.

21 Partially supported by Comunidad Autónoma de Madrid, Spain.

22 Supported by Fundación Banco Exterior.

23 Present address: SSC, Dallas, TX, USA.

24 Present address: Department of Energy, Washington.

25 Present address: Centre for Subatomic Research, University of Alberta, Canada and TRIUMF, Vancouver, Canada.

26 Present address: DESY, Hamburg, FRG.

27 Now with McKinsey Consultants, Sidney, Australia.

28 On leave and supported by DESY 1992-93.

29 On leave and supported by DESY 1991-92.

30 Permanent address Dipartimento di Fisica, Universita di Salerno, Salerno, Italy.

31 Present address: Hiroshima National College of Maritime Technology.

32 Presently on leave of absence at NIKHEF Amsterdam. 


\section{Introduction}

Results from inclusive electron-proton scattering have been seminal in establishing our present picture of hadronic structure [1]. Precise measurements of electron, neutrino and muon scattering from both nucleon and nuclear targets have allowed detailed determinations of the parton distributions. The results also provide a major testing ground for Quantum Chromodynamics, both in studies of the evolution of the structure functions with $Q^{2}$, the square of the momentum transfer, as well as in comparisons of data to the predictions of various sum rules. With the first operation of the electron-proton colliding-beam facility, HERA, at DESY, such experiments can be extended into a new range of the kinematic variables $Q^{2}$ and Bjorken $x$ [2]. Results from the $\mathrm{H} 1$ Collaboration have been presented in ref. [3]. The results given in this letter come from the first month of running and cover the $Q^{2}$ range from a few $\mathrm{GeV}^{2}$ up to $\sim 1000 \mathrm{GeV}^{2}$. Due to the large centre of mass energy, $\sqrt{s}=296 \mathrm{GeV}$, the events occur at low values of Bjorken $x$ extending the range studied by previous experiments by two orders of magnitude, to $x \sim 10^{-4}$ at $Q^{2} \sim 10 \mathrm{GeV}^{2}$. The data, therefore, explore nucleon structure in a new kinematic regime.

\section{Kinematics of deep inelastic scattering at HERA}

To describe the kinematics of the events in the neutral current, deep inelastic scattering (DIS) reaction

$\mathrm{ep} \rightarrow \mathrm{eh}$,

where $\mathbf{h}$ is the final hadronic system, it is conventional to use the Lorentz scalars $y$ (the normalised energy transfer to the proton in its rest frame), $Q^{2}$ and $x$. These variables may be determined from measurements of the electron in the laboratory frame:

$y=1-\frac{E_{\mathrm{e}}^{\prime}}{2 E_{\mathrm{e}}}\left(1-\cos \theta_{\mathrm{e}}\right)$,

$Q^{2}=2 E_{\mathrm{e}} E_{\mathrm{e}}^{\prime}\left(1+\cos \theta_{\mathrm{e}}\right)$,

and the relation

$x=\frac{Q^{2}}{s y}$.
In these equations, $E_{\mathrm{e}}$ is the energy of the incident electron, and $\theta_{\mathrm{e}}$ and $E_{\mathrm{e}}^{\prime}$ refer to the polar angle and energy of the scattered electron. Angles are defined relative to the proton beam direction which is also the direction of the positive $Z$ axis. The $X$ axis points to the centre of the HERA ring.

Since the hadronic final state is also measured, the kinematic properties of the DIS events can be determined in several ways [4]. In particular, in the socalled double angle (DA) method, the $y, Q^{2}$ and $x$ variables can be measured using only the angle of the scattered electron and an angle that characterises the final state hadronic system $\left(\gamma_{\mathrm{h}}\right)$. In the naïve quarkparton model $\gamma_{\mathrm{h}}$ would be the scattering angle of the struck quark. In general, the angle $\gamma_{\mathrm{h}}$ is given by fourmomentum conservation in terms of the transverse and longitudinal energy flow of the hadronic system $h$ as

$$
\begin{aligned}
& \cos \gamma_{\mathrm{h}} \\
& =\frac{\left(\sum_{\mathrm{h}} p_{X}\right)^{2}+\left(\sum_{\mathrm{h}} p_{Y}\right)^{2}-\left(\sum_{\mathrm{h}}\left(E-p_{Z}\right)\right)^{2}}{\left(\sum_{\mathrm{h}} p_{X}\right)^{2}+\left(\sum_{\mathrm{h}} p_{Y}\right)^{2}+\left(\sum_{\mathrm{h}}\left(E-p_{Z}\right)\right)^{2}},
\end{aligned}
$$

where $p_{X}$ and $p_{Y}$ are the transverse momentum components, $p_{z}$ the longitudinal component and $E$ the energy of the particles belonging to the system $h$. As the double angle method relies on ratios of energies it is less sensitive to a scale error in the energy measurement of the final state particles.

The variable $y$ can also be defined from the hadronic system $h$ as

$y=\frac{\sum_{\mathrm{h}}\left(E-p_{z}\right)}{2 E_{\mathrm{e}}}$.

This expression is exact when the sum runs over all the particles of $h$. Since particles emitted close to the forward direction contribute little to the above sum, it is a good approximation in the ZEUS experiment, which covers the full solid angle except for a small inactive region to accommodate the beampipe. When measured in this way, the variable is called $y_{\mathrm{JB}}$ (JacquetBlondel method [5]).

In the analysis, the sum over particles in equations (5) and (6) is replaced by the sum over all calorimeter cells: $E$ is now the energy measured in the cell and $p_{X}, p_{Y}$ and $p_{Z}$ are the measured energy multiplied by 
appropriate angular factors. The angles are measured from the ep interaction point to the centre of the energy deposit in each cell. Monte Carlo studies show that the error introduced by these approximations is small compared to the effect of the finite energy resolution of the calorimeter.

\section{The ZEUS detector}

ZEUS is a general purpose, magnetic detector with a tracking region surrounded by a high resolution calorimeter followed in turn by a backing calorimeter and a muon detection system [6].

The high resolution, uranium scintillator calorimeter (CAL) [7] is the principal component used in this analysis. It is divided into three sections, the forward (FCAL), barrel (BCAL) and rear (RCAL) calorimeters. For normal incidence, the depth of the CAL is 7 interaction lengths in FCAL, 5 in BCAL and 4 in RCAL.

The relative thicknesses of uranium and scintillator in the layer structure were chosen to give equal calorimeter response to electrons (or photons) and hadrons. Under test beam conditions, the energy resolution for electrons was measured $[7,8]$ to be $\sigma(E) / E=0.18 / \sqrt{E}$ and for hadrons $\sigma(E) / E=$ $0.35 / \sqrt{E}$, where $E$ is in $\mathrm{GeV}$.

Scintillator tiles form towers in depth that are read out on two sides through wavelength shifter bars, light guides and photomultipliers (PMTs). The towers are longitudinally segmented into electromagnetic (EMC) and hadronic (HAC) cells. The towers in FCAL and BCAL each have two HAC cells whereas those in RCAL have one. The depth of the EMC cells is 25 radiation lengths corresponding to one interaction length. Characteristic transverse sizes are $5 \mathrm{~cm} \times 20 \mathrm{~cm}$ for the EMC cells of FCAL and BCAL and $10 \mathrm{~cm} \times 20 \mathrm{~cm}$ for those in the RCAL. The HAC cells are typically $20 \mathrm{~cm} \times 20 \mathrm{~cm}$ in the transverse dimension. The towers are read out by a total of 11836 PMTs.

The construction minimises the possibility for particles from the interaction point (IP) to propagate down the boundaries between modules. Holes of $20 \mathrm{~cm} \times 20 \mathrm{~cm}$ in the centre of FCAL and RCAL are required to accommodate the HERA beam pipe. The resulting solid angle coverage is $99.7 \%$ of $4 \pi$.
During the running period more than $97 \%$ of all PMTs were in operation. Because each cell is read out by two PMTs there were never more than six cells $(0.1 \%)$ without operational readout. The calorimeter readout provides an energy and a time measurement for every PMT. The techniques used for dealing with the HERA interbunch time of $96 \mathrm{~ns}$ are discussed in ref. [9].

The gains of the PMTs were set from test data, in particular from the extensive measurements of the CAL modules made at CERN [7] and Fermilab [8]. These tests showed that the PMT gains can be set to sufficient precision from the signal that comes from the natural radioactivity of the uranium. The overall calibration, which was stable at the $0.2 \%$ level, was monitored frequently, using firstly the uranium signal, secondly charge injection to the input of the readout circuits and finally light from light emitting diodes or lasers, injected in front of the photocathodes of each PMT. The latter was also used to set the timing of all of the PMTs to the $1 \mathrm{~ns}$ level of precision.

The calorimeter noise, which is dominated by the uranium radioactivity, is small: the RMS width of the pedestal was typically $10 \mathrm{MeV}$ for an EMC PMT and $15 \mathrm{MeV}$ for a HAC PMT. Coherent noise between different cells was negligible. Although the noise for an individual PMT is small, the large number of cells in the CAL means that some can have noise pulses that are large enough to influence the measurement of the kinematic variables at low values of $y_{\mathrm{JB}}$ (see section 7).

Charged particles were measured by a jet-type drift-chamber (CTD) surrounding the beam pipe. The CTD consists of 72 cylindrical drift chamber layers organised into nine superlayers. The wires in the even-numbered superlayers have small stereo angles to allow a three-dimensional reconstruction of tracks. In the other, axial, superlayers the wires are parallel to the $Z$ axis. Only the inner three axial superlayers were instrumented for this data taking period using a $Z$-by-timing readout $[10]$ with single wire resolutions of $\sigma_{z}=4.1 \mathrm{~cm}$ and $\sigma_{r \phi}=1 \mathrm{~mm}$.

$A$ thin superconducting solenoid inside the calorimeter surrounds the tracking detectors. It has an inner diameter of $172 \mathrm{~cm}$, an overall length of $280 \mathrm{~cm}$ and a thickness of 0.9 radiation lengths. For the present data it provided a central magnetic field of $1.43 \mathrm{~T}$. 
Two small lead-scintillator sandwich counters (C5) partially surround the beam pipe at the rear of the RCAL. These counters were used to signal backgrounds produced by the incoming proton beam and to measure the timing and longitudinal spread of both the proton and electron beams of HERA. Two layers of scintillation counters mounted on either side of an iron vetowall, situated upstream of the detector, were also used to signal background particles.

The ep luminosity was measured from the rate of the bremsstrahlung process

$\mathrm{ep} \rightarrow \mathrm{ep} \gamma$,

by tagging the final state electron and photon in coincidence [6]. The luminosity monitor was also used to tag photons from initial state radiation and electrons from photoproduction processes. The integrated luminosity for the present data is $2.1 \mathrm{nb}^{-1}$ with a systematic uncertainty of $\pm 14 \%$ arising from rate dependent random vetos of the luminosity trigger, the subtraction of the beam gas background and the dependence of the acceptance on the position and angle of the electron beam.

\section{Monte Carlo simulation}

The detector acceptance and performance were studied extensively with a Monte Carlo simulation of the experiment. Neutral current DIS events with $Q^{2} \geqslant 2 \mathrm{GeV}^{2}$ were generated. The event generator used was HERACLES 4.1 [11] which includes electroweak radiative corrections to first order. The QCD cascade was simulated using the colour dipole model [12] as implemented in ARIADNE 3.0 [13] and the soft hadronisation process was described by the Lund string model, using JETSET 6.3 [14]. Various parametrisations of the parton densities were implemented: unless otherwise stated, the comparisons shown use the MRSD0 [15] set that fits the latest data from the NMC Collaboration [16] extending down to $x=0.008$ at a $Q^{2}$ value of about $4 \mathrm{GeV}^{2}$ as well as new data from the CCFR Collaboration [17].

The detector simulation, which is based on the general purpose programme GEANT 3.13 [18], incorporates our current knowledge of the experimental environment and trigger. The description of the responses of the various detector components was tuned to reproduce test data. The CAL noise was simulated according to the measured noise distributions.

\section{Data acquisition and trigger}

HERA has 220 bunches. For this first running period, in general, only 9 consecutive bunches were filled with electrons and protons. In addition, one electron or proton bunch circulated without colliding with a respective proton or electron bunch. These unpaired bunches allow the study of various backgrounds. The electron and proton currents were typically 1-2 mA. Since the electron bunch length is small the size of the interaction region $\left(\sigma_{z} \sim 20 \mathrm{~cm}\right)$ is determined by the proton bunch length.

The data reported here were collected using a trigger relying solely on the information from the calorimeter and the beam-monitor counters (C5). At the present luminosity, of the three available trigger levels, only the first (FLT) and third (TLT) level trigger stages were needed to produce an acceptable trigger rate. The TLT uses a farm of RISC processors.

For triggering, the calorimeter readout was grouped into 448 non-overlapping trigger towers having a transverse size of about $20 \mathrm{~cm} \times 40 \mathrm{~cm}$ constructed by summing the signals from adjacent calorimeter readout towers. A first-level trigger was issued whenever the energy sum in any of the EMC (or FCAL HAC) trigger towers exceeded a programmable threshold. The events of interest here were predominantly triggered by signals in the EMC sections of the RCAL and BCAL where the thresholds were set at $2.5 \mathrm{GeV}$, except the towers adjacent to the RCAL beam pipe where a $10 \mathrm{GeV}$ threshold was used. First level triggers were vetoed if their signals were in coincidence with a signal from the $\mathrm{C} 5$ counters that was in time with the proton beam. The loss of events due to this veto being in coincidence with a DIS trigger was less than $10^{-3}$. The total first level trigger rate was $8 \mathrm{~Hz}$. The TLT rejected proton beam induced background events by a loose cut on the average FCAL and RCAL times. About $35 \%$ of the triggers were rejected by this cut. It also removed triggers in which an electrical discharge in only a single PMT caused the trigger conditions to be satisfied. The output trigger rate 
was about $3 \mathrm{~Hz}$ for $1 \mathrm{~mA}$ of circulating protons. The resulting dead-time was less than $0.1 \%$.

The trigger acceptance for neutral current events was studied by Monte Carlo techniques. In general it increases with increasing $Q^{2}$ and is flat in $x$. For $x>$ $3 \times 10^{-4}$ and $Q^{2}>8 \mathrm{GeV}^{2}$ it exceeds $97.5 \%$.

The efficiency of the trigger hardware was monitored with selected data samples and with charge injection into the front-end electronics of the calorimeter; less than $3 \%$ of the trigger towers gave no signal. For the interval $10 \mathrm{GeV}^{2}<Q^{2}<100 \mathrm{GeV}^{2}$ more than $95 \%$ of all events satisfied the RCAL EMC trigger, where all channels were active.

The sensitivity to defects of individual trigger channels was reduced by the fact that a single event can exceed more than one of the trigger thresholds so that the overall efficiency of the hardware was greater than $99 \%$ in all regions of $x$ and $Q^{2}$. The online thresholds are low compared to the typical energies of the scattered electrons, thus the trigger calibration scale error $(10 \%)$ contributed an uncertainty of only $\pm 1 \%$ to the trigger acceptance for $Q^{2}>10 \mathrm{GeV}^{2}$.

\section{Event reconstruction and selection}

In the off-line analysis, hits in the CTD were used to reconstruct charged tracks which in turn allowed a vertex reconstruction: a valid vertex required at least two well measured tracks. Monte Carlo studies indicate that the vertex resolution in $Z$ is $\sigma_{Z}=4 \mathrm{~cm}$ compared to an rms length of the interaction region of $\pm 20 \mathrm{~cm}$. The measured vertex is used to determine the angular coordinates of the energy deposits in the calorimeter. In cases where a vertex could not be reconstructed, all three spatial coordinates were set to zero. Since the vertex resolution in the $X$ and $Y$ coordinates is about $0.6 \mathrm{~cm}$, significantly larger than the beam spread of $0.1 \mathrm{~mm}$, these coordinates of the vertex were set to zero when angles were calculated.

In the data analysis, EMC cells with an energy deposit of less than $60 \mathrm{MeV}$ and HAC cells with less than $110 \mathrm{MeV}$ were not considered. Cells were also ignored if an individual PMT contained less than $30 \mathrm{MeV}$ for the EMC or $45 \mathrm{MeV}$ in the case of the HAC. These cuts were chosen in order to minimise the influence of noise on the measurement of the kinematic variables.
The key signature of neutral current events is the presence of a scattered electron in the final state. Several electron-identification algorithms have been developed. All of them are based on the small lateral and longitudinal spreads in energy deposition expected for electromagnetic showers. Hadrons typically produce wide showers that extend deep into the calorimeter. The algorithms also account for the increase in the longitudinal spread when the electron impinges on the cracks between CAL modules and the increase in the lateral spread caused by preshowering in the inactive material in front of the CAL.

The discrimination between an electron (or photon) and a hadron is based on the energy weighted mean radius of the EMC energy depositions within an inner cone and the EMC energy as well as the ratio of HAC to EMC energy in an outer cone. After identification, all calorimeter energy within the outer cone was summed to give the electron energy. When applied to neutral current events generated by Monte Carlo techniques, the selected algorithm correctly identified more than $98 \%$ of all scattered electrons with an energy of more than $5 \mathrm{GeV}$ that were inside the fiducial volume of the detector.

The position of the electron was measured from the sharing of the energy between the two PMTs viewing the cell containing the maximum energy and, in the orthogonal direction, by the energy sharing between this cell and those adjacent. The position resolution in $X(Y)$ was typically $1.2(2.1) \mathrm{cm}$ for the RCAL leading to an average resolution in polar angle of $\sigma_{\theta_{\mathrm{e}}}=$ $0.5^{\circ}$.

The $1.05 \times 10^{6}$ events accepted by the trigger were passed through a series of filters to select the small number of neutral current events that were contained in the sample. In order to retain redundancy the first stage of the filter used the combination of two independent selections. The first one relied on the EMC trigger being satisfied in RCAL (or BCAL). The second selection required an identified electron with an energy of more than $4 \mathrm{GeV}$. These selections retained $1.4 \%$ of the events.

An algorithm based on the topology of cells in a single calorimeter section was used to reject cosmic rays. The efficiency of this algorithm was $80 \%$. Monte Carlo studies were used to ensure that no neutral current events were lost by applying these cuts.

After this preselection 12637 events remained. 

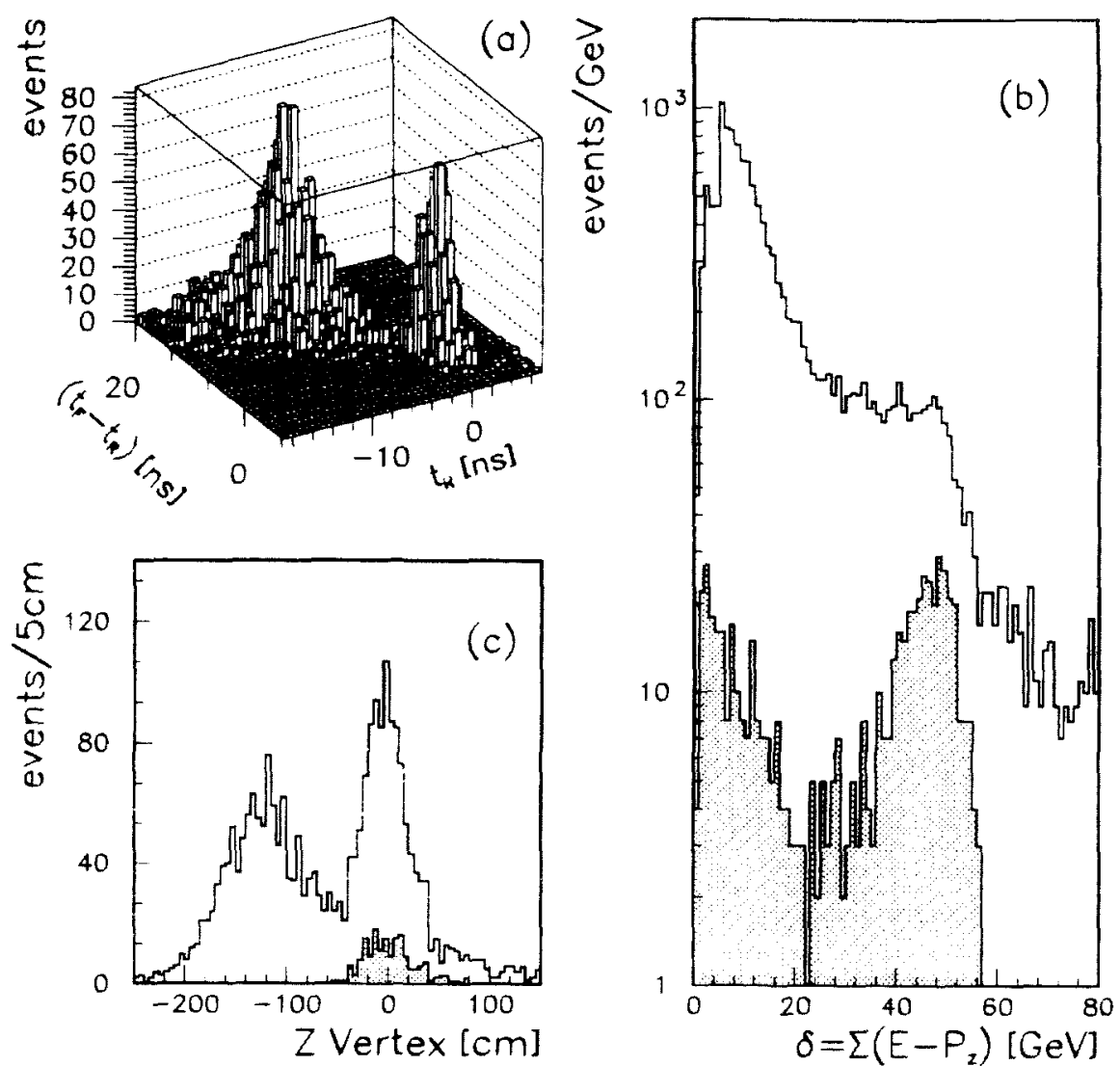

Fig. 1. (a) Distribution of preselected events in (FCAL-RCAL) and RCAL times. The peak at the origin which is well separated from the background contains the events originating from the IP. (b) The distribution of $\delta$ at the preselection level. The shaded area shows the event distribution after all the cuts except that on $\delta$ itself. (c) The $Z$ distribution of event vertices at the preselection level. The shaded area shows the vertex distribution of the final event sample with $y_{\mathrm{JB}}>0.02$.

A large fraction of the beam induced background comes from proton interactions outside the detector region and was removed by a more refined cut on the average RCAL and FCAL times. They were calculated as an energy weighted mean of the times, $t_{i}$, of those PMTs that had an energy $E_{i}>200 \mathrm{MeV}$. The calibration was chosen such that ep events originating at the nominal interaction point give $t_{i}=0$. The resulting RCAL time distribution has a spread of $\sigma=$ $0.84 \mathrm{~ns}$. The FCAL distribution is somewhat wider (1.2 ns), reflecting the length of the proton bunch in HERA. Fig. 1a shows the event distribution in a plot of $\left(t_{\mathrm{F}}-t_{\mathrm{R}}\right)$ versus $t_{\mathrm{R}}$ for events that give a time measurement both in FCAL and RCAL ( $41 \%$ of the events). Two clear, separated clusters of events can be seen. The backgrounds have RCAL times that are early compared both to the FCAL time and to the absolute time for signals from ep interactions at the IP. When $t_{\mathrm{F}}$ and $t_{\mathrm{R}}$ were measured, an event was accepted if $-6 \mathrm{~ns}<\left(t_{\mathrm{F}}-t_{\mathrm{R}}\right)<0.5 t_{\mathrm{R}}+6 \mathrm{~ns}$ and $\left|t_{\mathrm{F}}\right|,\left|t_{\mathrm{R}}\right|<$ $6 \mathrm{~ns}$. All but three of the final DIS events have a measured time in both FCAL and RCAL. The resulting acceptance loss of DIS events was less than $0.5 \%$.

One component of the remaining background comes from the interaction of the electron beam with the residual gas. Events originating from the unpaired electron bunch but subject to an RCAL time cut, show little or no energy deposit in the FCAL. Requiring an FCAL energy of more than $1 \mathrm{GeV}$ removes all of these events while Monte Carlo studies 
indicate that only $\sim 3 \%$ of the DIS events are lost, predominantly at low $y$.

Events containing a vetowall signal and those with an energy in the electron calorimeter of the luminosity detector of greater than $5 \mathrm{GeV}$ were rejected.

A cut of $5 \mathrm{GeV}$ on the energy of the candidate electron was imposed to reduce the background from falsely identified hadrons and a fiducial cut around the beam pipe of $(|X|,|Y|>16 \mathrm{~cm})$ at the face of the RCAL was imposed to allow an accurate position and energy measurement of the electron.

An important variable that characterises the events is the quantity $\delta$ defined by

$\delta=\sum_{i}\left(E-p_{z}\right)_{i}$,

where the sum runs over all measured particles. For ep events where all final state particles are measured, $\delta=$ $2 E_{\mathrm{e}}$. Undetected particles which are emitted down the forward beam pipe give a negligible loss. Initial state radiation moves events to lower values and measurement errors lead to a broadening of the distribution.

Different final states produce different $\delta$ distributions, so that it is a good discriminator against background processes. Photoproduction events in which the scattered electron stays in the beam pipe, but which are selected because an energy deposit has been falsely interpreted as the scattered electron, will predominantly give low values of $\delta$. Background events coming from interactions of the proton beam also have small values of $\delta$.

Fig. $1 \mathrm{~b}$ shows the distribution in $\delta$ calculated from the energies measured in the CAL towers. The preselected events, shown in the upper curve, have a broad shoulder at the position expected for DIS events superimposed on a large falling background. Most of this background is removed by the timing and FCAL energy cuts and a clear two peak structure then appears, as seen in the shaded area. The accumulation of events at low values of $\delta$ is well separated from the distribution of DIS events and was removed by the selection: $35 \mathrm{GeV}<\delta<60 \mathrm{GeV}$. The lower value is in the region separating the two peaks and the upper cut allows for resolution smearing.

The effect of each of the rejections was carefully studied, in particular by a visual inspection of a sample of the rejected events. In a scan of the finally selected event sample, five remaining cosmic and beam muons were identified and removed. After these selections 305 events remained. None came from an unpaired electron or proton bunch.

No requirement has been placed on the vertex reconstructed from the CTD tracks. Although most of the electrons are outside of the acceptance of the CTD, an event vertex can be reconstructed from the charged hadrons in $58 \%$ of the events. A vertex was reconstructed in 55\% of the Monte Carlo events, in satisfactory agreement with the data. The major contribution to this inefficiency is due to the fact that events at low $y$ show no activity in the CTD. In the final event sample, with $y_{\mathrm{JB}}>0.02,80 \%$ of the events have a reconstructed vertex. The distribution of reconstructed $Z$ vertices for the preselected events is shown in fig. 1c. Two clear peaks can be seen, the one at negative $Z$ values from background processes and the accumulation near $Z=0$ coming from ep scattering (e.g., DIS and photoproduction). The vertex distribution for the final event sample is shown shaded. The width is consistent with that expected from the known proton bunch length.

The photoproduction background was estimated by generating photoproduction events using PYTHIA [19], and passing them through the complete analysis chain. This study predicted that for a total $\gamma \mathrm{p}$ cross section of $154 \mu \mathrm{b}[6]$ and our integrated luminosity the data sample is expected to contain four such events, approximately one quarter of which should have an electron in the luminosity monitor with energy greater than $5 \mathrm{GeV}$. Our observation of one such electron is consistent with this Monte Carlo estimate. We therefore take this background to be $4 \pm 2$ events.

\section{Results}

Fig. 2 compares some properties of the selected events with the predictions of the Monte Carlo simulation. The data are shown as points with statistical errors and the simulation as the shaded histograms. The distributions in electron energy and angle are shown in figs. $2 a$ and $b$. While the angular distribution, mostly a consequence of the $1 / Q^{2}$ of the photon propagator, is well described by the Monte Carlo, the position of the peak in the measured electron energy spectrum is shifted to lower values than expected. 

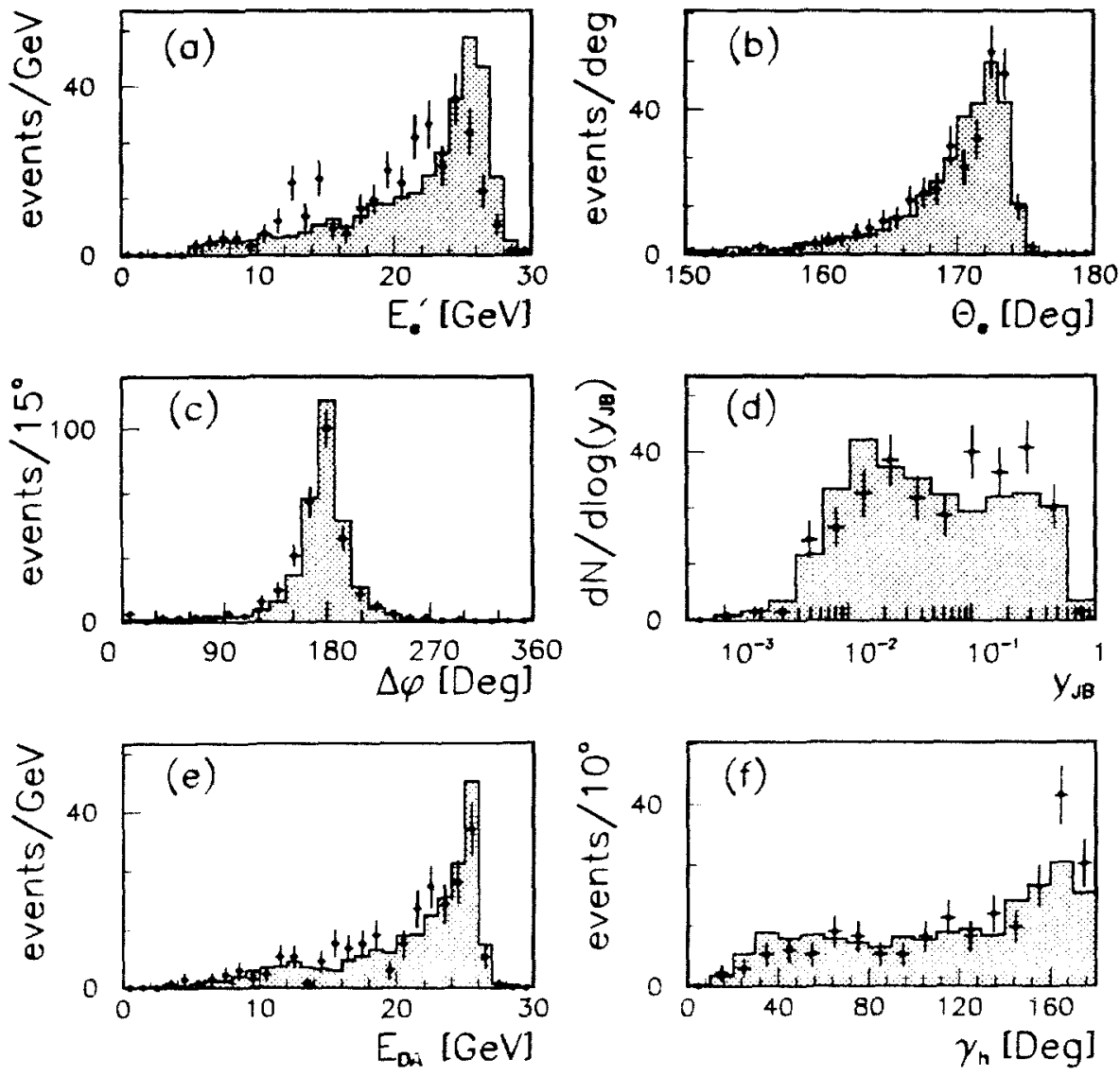

Fig. 2. Comparisons of the data with the Monte Carlo simulation: (a) electron energy, (b) electron angle, (c) $\Delta \phi$, (d) $y_{\mathrm{JB}}$, (e) electron energy calculated from $\theta_{\mathrm{e}}$ and $\gamma_{\mathrm{h}}$, and (f) hadron angle. The data are shown as the points with statistical errors and the Monte Carlo simulation as the histograms. A cut of $y_{\mathrm{JB}}>0.02$ has been applied to plots (e) and (f).

We attribute this effect primarily to present limitations of the geometry modelling, especially of inactive material between the interaction point and the front of the calorimeter, in the Monte Carlo code. Despite this limitation, the overall characteristics of the events are well reproduced. For example, the electron and hadron energy flows are back to back in the transverse plane, as seen in fig. $2 \mathrm{c}$ which shows the distribution in the difference between the azimuthal angles $(\phi)$ of the electron and the hadronic system.

Fig. $2 \mathrm{~d}$ shows the distribution in $y_{\mathrm{JB}}$. There is good agreement between data and Monte Carlo even at the smallest values of $y_{\mathrm{JB}}$, which are sensitive to $\mathrm{CAL}$ noise. For the remaining distributions events with $y_{\mathrm{JB}}<0.02$ are removed since the resolution in kinematic variables deteriorates significantly in this re- gion and the measurements suffer from systematic biases coming from the effects of noise and the finite segmentation of the calorimeter.

The energy of the final state electron can also be calculated from the angles $\theta_{\mathrm{e}}$ and $\gamma_{\mathrm{h}}$ using the equation

$E_{\mathrm{DA}}^{\prime}=2 E_{\mathrm{c}} \frac{\sin \gamma_{\mathrm{h}}}{\sin \gamma_{\mathrm{h}}+\sin \bar{\theta}_{\mathrm{e}}-\sin \left(\theta_{\mathrm{e}}+\gamma_{\mathrm{h}}\right)}$.

The distributions in $E_{\mathrm{DA}}^{\prime}$ are shown in fig. 2e. The data exhibit a kinematic peak in agreement with the simulation. The distribution in $\gamma_{\mathrm{h}}$, shown in fig. $2 \mathrm{f}$, also gives satisfactory agreement with the Monte Carlo simulation.

The variables $x$ and $Q^{2}$ are now determined from $\theta_{\mathrm{e}}$ and $\gamma_{\mathrm{h}}$ and fig. $3 \mathrm{a}$ shows the distribution of events in the $\left(x, Q^{2}\right)$ plane. The lines of constant $y$ values 



Fig. 3. (a) The distribution of events in the $\left(x, Q^{2}\right)$ plane. (b) The $Q^{2}$ distribution of the events. (c) The $x$ distribution of the events. The Monte Carlo predictions, shown is the shaded histograms, are calculated from the extrapolation of the MRSD0 parametrisation. (d) The measured differential Born cross sections, in the selected $\left(x, Q^{2}\right)$ ranges indicated in (a), compared to the extrapolations of three parton density functions: MTB1, MTB2 and MRSD0.

of $0.01,0.1$ and 1.0 are indicated. The projections of the data in $x$ and $Q^{2}$, shown in figs. $3 \mathrm{~b}$ and $3 \mathrm{c}$, are in satisfactory accord with the Monte Carlo simulation. Although the events are predominantly at low values of $Q^{2}$, overlapping the $Q^{2}$ range of previous experiments, the present data extend about two orders of magnitude lower in $x$ than has previously been studied.

In measuring the cross sections it is important to choose regions in the $\left(x, Q^{2}\right)$ plane where the acceptance is both uniform and high. Three $Q^{2}$ bins: $10<$ $Q^{2}<20,20<Q^{2}<40,40<Q^{2}<100 \mathrm{GeV}^{2}$ and two $x$ ranges: $0.0005<x<0.0015$ and $0.0015<$ $x<0.008$ were chosen as indicated in fig. $3 \mathrm{a}$. These choices were dictated by the resolution which is bet- ter than $40 \%$ for $x$ and $25 \%$ for $Q^{2}$. In these bins the acceptance varies from $70 \%$ for the low $Q^{2}$ bin, which is affected by the $y_{\mathrm{JB}}>0.02$ cut, to $93 \%$ for the highest $Q^{2}$ bin. The total corrections for acceptance and migration due to the finite $x$ and $Q^{2}$ resolution were less than $20 \%$ and were insensitive to the choice of the parton density distribution. The data were corrected for these effects. In order to take into account QED radiative effects a conversion was made to the lowest order (Born) cross section using the MRSD0 parton density distribution. This was done by calculating the correction factors both with the radiative effects turned on and with them turned off. The statistical errors of the present data preclude an iterative procedure for this conversion. 
Fig. 3d shows the cross section values. The lines are the Born cross sections calculated from extrapolations of three parton density sets, MRSD0, MTB1 and MTB2 [20], into this new kinematic region. In these calculations, the contribution from the longitudinal structure function, $F_{\mathrm{L}}$, was ignored. Although QCD predicts that $F_{\mathrm{L}}$ is large in some regions of the kinematics, the effect is at most $2 \%$ in the $\left(x, Q^{2}\right)$ regions used in determining the cross sections. This effect is included in the overall systematic error. There is good agreement with all three parametrisations at the present precision of the experiment.

The inner error bars shown on the cross sections are statistical, while the outer error includes a linear addition of the estimated systematic effects that we now discuss. The errors do not include the overall scale uncertainty of $14 \%$ from the luminosity measurement.

The sensitivity of the cross sections to the cuts was checked by relaxing them individually within reasonable limits. The cross sections were also measured using other techniques to determine $x$ and $Q^{2}$. In the selected $\left(x, Q^{2}\right.$ ) bins, $80 \%$ of the events have a reconstructed vertex. Cross sections determined from this fraction alone are in excellent agreement with those measured from the full sample. The error introduced by using the MRSD0 parametrisation for the extraction of the Born cross section was estimated by also determining the conversion using the MTB1 and MTB2 parton density distributions. The resulting systematic errors are between $15 \%$ and $25 \%$, depending on the $Q^{2}$ value, dominated by the uncertainty in the conversion to the Born cross section.

\section{Conclusions}

This paper presents our first study of neutral current deep inelastic ep scattering. The events were observed in a full solid angle detector so that both the electron and the secondary hadrons could be used to characterise the events. The ZEUS detector, in particular the high resolution calorimeter, allows the selection of DIS events with very little background. The measurement of the scaling variables can be made using several techniques. Within the statistics of the present analysis, the distributions in the scaling variables and the cross sections in selected $\left(x, Q^{2}\right)$ bins are in accord with extrapolations of current parametrisations of parton densities. In the measured $Q^{2}$ range the data extend to values of $x$ which are two orders of magnitude smaller, than previously reached by fixed target experiments.

\section{Acknowledgement}

We thank the DESY Directorate for their strong support and encouragement. The experiment was made possible by the inventiveness and the diligent efforts of the HERA machine group who succeeded in making HERA run in a very short time.

The design, construction and installation of the ZEUS detector has been made possible by the ingenuity and dedicated effort of many people from inside DESY and from the home institutes who are not listed as authors, especially the on-site efforts of T. Winch, J. Hauschildt and K. Westphal. Their contributions are acknowledged with great appreciation.

This work has been supported by grants from the Natural Sciences and Engineering Research Council, and the FCAR of Quebec, Canada, by the German Federal Ministry for Research and Technology (BMFT), by the Deutsche Forschungsgemeinschaft (DFG), by the Italian National Institute for Nuclear Physics (INFN), by the Japanese Ministry of Education, Science and Culture (the Monbusho) and its grants for Scientific Research, by the Netherlands Foundation for Research on Matter (FOM), by the Polish Government and Ministry of Education Research Programs, by the Spanish Ministry of Education and Science through funds provided by CICYT, by the UK Science and Engineering Research Council, by the MINERVA Foundation, by the Israel Academy of Science, by the Israel Department of Energy, by the German Israeli Foundation, by the US Department of Energy and by the US National Science Foundation.

\section{References}

[1] E.D. Bloom et al., Phys. Rev. Lett. 23 (1969) 930.

[2] Results from a preliminary analysis of this data as well as the results from the $\mathrm{H} 1$ detector were presented at the 26th Intern. Conf. on High energy physics (Dallas, 1992 ) by B. Löhr (ZEUS) and F. Eisele (HI). 
[3] T. Ahmed et al., H1 Collab., DESY 92-164, submitted to Phys. Lett. B.

[4] S. Bentvelsen et al., Proc. Workshop on Physics at HERA vol. 1, (1992) 23.

[5] F. Jacquet and A. Blondel, Proc. of the study for an ep facility for Europe, DESY 79/48 (1979) 391.

[6] ZEUS Collab., M. Derrick et al., Phys. Lett. B 293 (1992) 465.

[7] M. Derrick el al., Nucl. Instrum. Methods A 309 (1991) 77;

A. Andresen et al., Nucl. Instrum. Methods A 309 (1991) 101.

[8] A. Bernstein et al., to be submitted to Nucl. Instrum. Methods;

I. Gialas, Proc. Second Intern. Conf. on Calorimetry for high energy physics (Capri, 1991) p. 162.

[9] A. Caldwell et al., Nucl. Instrum. Methods A 321 (1992) 356.

[10] N. Harnew et al., Nucl. Instrum. Methods A 279 (1989) 290;

B. Foster et al., Proc. 3rd Intern. Conf. on Advanced technology and particle physics (Villa Omo, Como, 1992), Oxford Univ. preprint OUNP-92-14, Nucl. Instrum. Methods (to be published).
[11] A. Kwiatkowski et al., Proc. Workshop on Physics at HERA vol. 3, (1992) 1294.

[12] B. Andersson et al., Z. Phys. C 43 (1989) 621.

[13] L. Lönnblad, Lund preprint LU TP-89-10.

[14] T. Sjöstrand, Comput. Phys. Commun. 39 (1986) 347; T. Sjöstrand and M. Bengtsson, Comput. Phys. Commun. 43 (1987) 367.

[15] A.D. Martin et al., Durham/RAL preprint, DTP-9216 and RAL-92-021, Phys. Rev. (to be published).

[16] NMC Collab., P. Amaudruz et al., Phys, Lett. B 295 (1992) 159.

[17] E. Oltman et al., Z. Phys. C 53 (1992) 51.

[18] R. Brun et al., preprint CERN DD/EE/84-1.

[19] H.U. Bengtsson and T. Sjöstrand, Comput. Phys. Commun. 46 (1987) 43;

T. Sjöstrand, Proc. Workshop on Physics at HERA vol. 3 (1992) 1405.

[20] J.G. Morfin and W.K. Tung, Z. Phys. C 52 (1991) 13.

[21] L. Gribov et al., Phys. Rep. 100 (1983) 1;

E. Levin and M. Ryskin, Nucl. Phys. B (Proc. Suppl) $18 \mathrm{C}(1990) 92$.

J. Bartels and J. Feltesse, Proc. Workshop on Physics at HERA vol. 1 (1992) 131. 